\title{
Trade Credit and Lending Strategy of Supply Chain with Information Asymmetry
}

Xiaofeng Xie ${ }^{1}$, Yang Yang $^{2}$, Zongfang Zhou ${ }^{1}$

${ }^{1}$ School of Management and Economics, University of Electronic Science and Technology of China, Chengdu 611731, China

${ }^{2}$ School of Economics Mathematics, Southwestern University of Finance and Economics, Chengdu 611130, China

\section{信息不对称下供应链商业信用与信贷策略研究}

\author{
谢小风 ${ }^{1}$, 杨扬 ${ }^{2}$, 周宗放 ${ }^{1}$ \\ ${ }^{1}$ 电子科技大学经管学院, 四川成都, 611731, 中国 \\ ${ }^{2}$ 西南财经大学经济数学学院, 四川成都, 611130, 中国
}

\begin{abstract}
In the symmetric case the lender and borrower has same information about buyer. While in the asymmetric case whether the buyer default is the private information of borrower. The theoretical framework we constructed in this study has focused on lending strategy of lender to the case when the buyer of borrower may default in the circumstance of supply chain. The information asymmetry is considered this framework and hence incentive compatibility constraint of borrower is necessary for lender to ensure desired effort made by borrower. We define the incentive compatibility constraint, which is technically the fundamental of our model in two case. The static comparison and numerical analysis of the model illustrate that the loan interest in symmetric case is significantly lower than it in asymmetric case. Default risk of buyer increases the cost of borrower compared to the case when buyer pays for sure and massive trade credit associated with higher loan interest. However the impact on loan amount of credit risk and default risk is more complex. It turns out that information asymmetry can be seen as a moderating factor in the impact on loan amount of default risk and credit risk.
\end{abstract}

Keywords: Information asymmetry, Supply chain, Trade credit, Lending strategy

\section{摘要}

在信息对称的情况下, 上游企业和银行关于下游企业 的行为具有一致的认识, 而在信息不对称的情况下, 上游企业具有更多的信息优势。本文构建了一个基本 理论框架刻画了当供应链下游企业对上游企业存在 违约风险时, 银行对上游企业的信贷策略。通过银行 对上游贷款人激励约束下的静态比较和数值分析, 结 果表明信息不对称将使得银行通过提高利率来弥补 可能的损失; 下游企业违约风险以及供应链商业信用 敞口的提升都会增加上游企业的资本成本。

关键词: 信息不对称, 供应链, 商业信用, 信贷策略

1. 引言

商业信用是指企业在商品交易时以商品形式 提供的信用, 典型形式为卖方向买方以㻌销方式提 供的信用。作为一种非常重要的筹资方式, 商业信 用不仅可以优化资金流，提高供应链的运作绩效， 还可以通过吸引企业增加订货, 迅速扩大上下游企 业的物流。

Wilson 和 Summers $(2002)^{[1]}$ 提出商业信用作为 一种有效内部融资资源, 被称为开放式账户融资, 在各种供应链中被广泛采用。尽管到目前为止, 与 商业信用有关的诸多问题尚未解决, 但针对商业信 用的某些具体问题, 许多学者进行了研究, 并已经 取得一些有价值的研究成果。一般地, 商业信用是 供应链企业的普遍融资来源, 尤其是在营运资金来 


\section{Risk Analysis and Crisis Response in Big Data Era (RAC-16)}

源有限, 银行融资难的前提下。Fishman 等 $(2003)^{[2]}$ 认为即使对那些经济高度发达的金融市场, 例如美 国, 信贷风险在短期融资中仍发挥着越来越重要的 作用, 更不用说欠发达国家了。

本文借鉴 Csokade $(2013)^{[3]}$ 的理论框架来刻画 信息不对称情况下供应链的商业信用。然而, 与他 们的工作相比, 本文有三方面不同: 首先, 运用平 均转移投资技术模型来描述风险项目收益的回报 规模; 其次, 简化了参数的设置, 以使研究目标和 研究重点集中于贷款人的贷款策略; 最后对模型进 行了数值仿真, 并做了详细分析。数值结果表明, 该模型具有较好的合理性和有效性, 能够为供应链 中银行制定信贷风险控制政策提供指导作用，同时 也能丰富商业信用的理论架构, 具有一定的现实意 义。

\section{2. 模型建立}

模型的基本框架假设如下: 供应链由上下游两个 企业构成。上游企业作为外部融资的借款者是风险中 性的。上游企业希望通过银行融资 $I$, 并将其投入到 一个风险项目上。该风险项目在未来的回报依赖于两 个方面的因素。第一是投资的金额。第二是上游企业 贷款以后为项目付出的努力 $\theta$ 。

结合这两方面的考虑, 本文采用 Koskela 和 Stenbacka (2000)的移动平均投资模型来对该项目的 投资回报以及其不确定性进行刻画。上游企业对于风 险项目付出的努力水平分别记为高努力水平 $\theta_{H}$ 和低 努力水平 $\theta_{L}$ 。从而, 投资金额为 $I$ 的风险项目的投 资回报 $\pi$ 分布于区间 $\left[0, I \theta_{i}\right]$, 其中 $i \in\{H, L\}$, $1<\theta_{L}<\theta_{H}$, 投资回报 $\pi$ 是一个满足条件密度函数为 $f\left(\bullet \mid I \theta_{i}\right)$, 分布函数为 $F\left(\bullet \mid I \theta_{i}\right)$ 的随机变量。

根据 Tirole (2006), 假设作为贷款人的供应链上 游企业初始资本为 0 , 由于投资的规模报酬, 上游企 业自然希望获得更多的贷款。进一步, 如果上游企业 选择低努力水平 $\theta_{L}$ 的时候, 假设其可以获得一个额 外的与项目总投资金额有关的回报 $B I$, 其中 $B>0$ 。 由于上游企业的回报同时也受到供应链下游企业违 约风险的影响,故在模型中需考虑下游企业违约概率 设下游企业履约概率为 $P$, 则违约概率为 $1-P$ 。根 据 Csoka 等 (2013)假设如果下游企业发生违约, 那 么将会损失风险项目回报中的 $\delta \in[0,1]$ 。这里 $\delta$ 正好 度量了供应链商业信用的敞口。记银行给上游企业的
利率为 $r$, 则如果风险项目的收益大于 $I(1+r)$, 则认 为项目成功; 否则视为项目失败。

与 Csoka 等(2013)一致, 本文重点研究两种情况 在信息对称的情况下, 无论是银行还是上游企业都不 知道下游企业违约前的行为。而在非对称情况下, 上 游企业知道下游企业是否会违约, 相较于银行而言, 上游企业拥有更多的信息优势。此信息优势在于上下 游企业间的业务交互作用。根据以上假设, 构造上游 企业的期望收益函数:

$$
E\left[\pi_{i}\right]=\beta Z_{i} B I+\int_{I(1+r) / \beta}^{I \theta_{i}}[\tau \beta-I(1+r)] f\left(\tau \mid I \theta_{i}\right) d \tau \quad(1)
$$

其中 $i \in\{H, L\} ; \beta=p+(1-p)(1-\delta) ; Z_{i}$ 为

影子变量, 当 $i=H$ 时, $Z_{i}=0$; 当 $i=L$ 时, $Z_{i}=1$.

假设模型中所有的参数都是共同信息。由于在低 努力水平下, 上游企业的个人收益是不能转移给银行 的, 那么仅仅当上游贷款企业作出高水平努力时, 才 可能偿还贷款, 也就是说 (2) 必须被满足

$$
\int_{0}^{I \theta_{L}}[\tau-I(1+r)] f\left(\tau \mid I \theta_{L}\right) d \tau<0
$$

在这样的假设下, 只有当借款人选择高努力水平 时, 项目才能成功。那么银行为了最大化自己的收益, 必将促使借款人必须选择高水平努力, 使自己收回贷 款。（2）式表明银行贷款的条件是上游企业满足激励 相容约束。当项目成功时, 银行会得到本金和利息 $I(1+r)$, 上游企业会得到除银行利息外的剩余收 益。

\section{1.信息对称的情况}

银行、上游企业与下游企业之间的博亦可以用图 1 进行阐述.

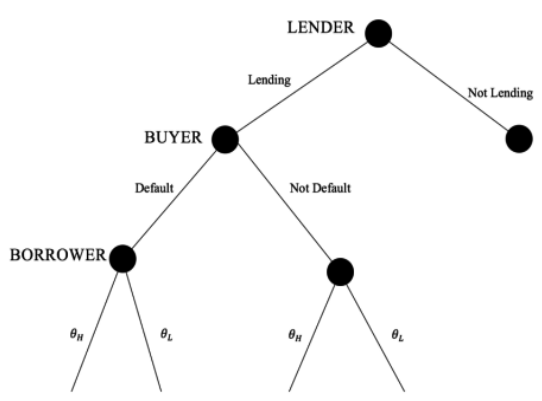

图 1：对称情况下的扩展式博亦 
Risk Analysis and Crisis Response in Big Data Era (RAC-16)

图 1 表明, 银行在不了解下游企业违约意愿的前 提下，先确定贷款金额和贷款利率，随后上游企业选 择它的努力水平, 最后下游企业做出决策。在本文中, 假定下游企业是否违约是外生的, 履约概率为 $P$, 违 约概率为 $1-P$ 。

银行提供的最优契约由银行的个体理性约束和上 游借款企业的激励相容约束共同决定。考虑到银行是 竞争性市场, 银行的个体理性约束保证了它的预期收 益比竞争均衡下的利润更具吸引力, 并能促使它借款 给上游企业。银行的竞争性均衡表示如下:

$$
\int_{0}^{I((+r) / \beta} \beta \tau f\left(\tau \mid I \theta_{H}\right) d \tau+\int_{I((+r) / \beta}^{I \theta_{H}} I(I+r) f\left(\tau \mid I \theta_{H}\right) d \tau=I \quad \text { (3) }
$$

无论下游企业是否违约, 激励相容约束对于上游 借款人选择更高的努力水平都是有必要的。考虑到借 款人在低努力水平下具有私人利益, 借款人的激励相 容约束应确保高努力水平所带来的利益超过低努力 水平下的收益。

上游贷款企业的激励约束为

$$
\begin{gathered}
\int_{I(1+r) / \beta}^{I \theta_{H}}[\beta \tau-I(1+r)] f\left(\tau \mid I \theta_{H}\right) d \tau \geq \\
B I+\int_{I(1+r) / \beta}^{I \theta_{L}}[\beta \tau-I(1+r)] f\left(\tau \mid I \theta_{L}\right) d \tau(4) \\
\text { 令 } \alpha=I(1+r) / \beta, \Delta \theta=\theta_{H}-\theta_{L} \text {, 则 (4) 可写为 } \\
\alpha \beta\left[F\left(\alpha \mid I \theta_{L}\right)-F\left(\alpha \mid I \theta_{H}\right)\right] \geq \\
I(B-\beta \Delta \theta)+\beta\left[\int_{\alpha}^{I \theta_{H}} F\left(\tau \mid I \theta_{H}\right) d \tau-\int_{\alpha}^{I \theta_{L}} F\left(\tau \mid I \theta_{L}\right) d \tau\right](5) \\
\text { 显然 }\left[\alpha, I \theta_{L}\right] \subset\left[\alpha, I \theta_{H}\right] \text {,从而有 } \\
\int_{\alpha}^{I \theta_{H}} F\left(\tau \mid I \theta_{H}\right) d \tau-\int_{\alpha}^{I \theta_{L}} F\left(\tau \mid I \theta_{L}\right) d \tau>0(6)
\end{gathered}
$$

不等式(5)可放缩为

$$
\alpha\left[F\left(\alpha \mid I \theta_{L}\right)-F\left(\alpha \mid I \theta_{H}\right)\right] \geq I(B-\beta \Delta \theta)
$$

\section{2. 信息不对称的情况}

在图 2 表示的博亦过程中, 供应链上游企业具有 信息优势, 即供应链上游企业在决策之前能够观测到 下游企业的履约行为。

与图 1 相比, 银行的地位没有改变, 它对对称和 非对称情况下下游企业的行为均一无所知。因此, 对 称与不对称情况下银行的个人理性约束都为等式 (3)。

这个时候银行的参与约束在分部积分以后变为:

$$
1-I r / \beta=\int_{\alpha}^{I \theta_{H}} F\left(\tau \mid I \theta_{H}\right) d \tau
$$

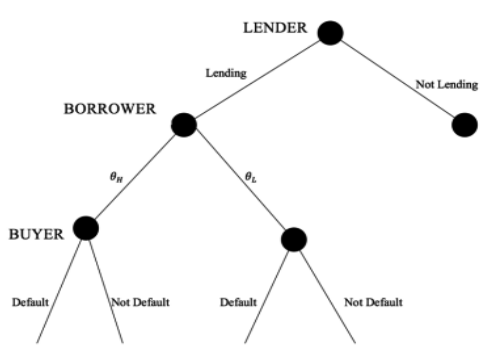

图 2 :不对称情况下的扩展式博亦

而借款人的激励相容约束与信息对称情况下不同, 不能用 (4) 表示。由于上游企业是在观察到下游企 业的行为后再做出决策, 无论下游企业是否违约, 银 行都应确保上游借款人选择更高水平的努力。于是上 游借款人的激励相容约束这时候由于博弯顺序的不 同变的不同。

如果下游企业履约, 则上游企业的激励相容约束 为

$I(\Delta \theta-B)+\beta\left[\int_{I(1+r)}^{I \theta_{H}} F\left(\tau \mid I \theta_{H}\right) d \tau-\int_{I(1+r)}^{I \theta_{L}} F\left(\tau \mid I \theta_{L}\right) d \tau\right]$ ( 9) 如果下游企业违约, 则上游企业的激励相容约束 为

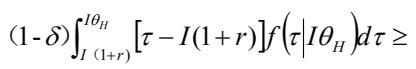

$B I+(1-\delta) \int_{I(1+r)}^{I \theta_{L}}[\tau-I(1+r)] f\left(\tau \mid I \theta_{L}\right) d \tau$

与（9）类似, 不等式 (10) 可表示为

$I\left(\Delta \theta-\frac{B}{1-\delta}\right) \geq \int_{I(1+r)}^{I \theta_{H}} F\left(\tau \mid I \theta_{H}\right) d \tau-\int_{I(1+r)}^{I \theta_{L}} F\left(\tau \mid I \theta_{L}\right) d \tau$ (11)

如上所述, 考虑到银行的预期收益, 无论下游企 业违约与否, 它应该确保借款人选择更高水平的努力。 这意味着不等式 (9) 和 (11) 应该满足。由于 $B(1-\delta)>B$, 不等式 (10) 满足时, 不等式 (11) 必然满足。因此, 信息不对称下的激励相容约束可表 示为不等式 (11). 
Risk Analysis and Crisis Response in Big Data Era (RAC-16)

\section{3. 静态比较和数值分析}

这一节分析了信息对称和信息不对称情况下银行 信贷决策行为的差异, 同时比较了银行信贷风险敞口 和下游企业违约概率所产生的影响。

第一步：固定贷款金额 $I$, 在信息对称情况下，综 合银行个人理性约束( 8$)$ 与不等式(7)可得到

$$
r \geq \beta I(B-\beta \Delta \theta)-1
$$

而在信息不对称情况下, 综合( 8 )与不等式 (12) 可得到

$$
r \geq \beta\left(1+\frac{I B}{1-\delta}-\Delta \theta\right)-\frac{\beta}{I} \int_{I(1+r)}^{I \theta_{L}} F\left(\tau \mid I \theta_{L}\right) d \tau
$$

在(1)中定义了 $\beta$, 且 $\beta<1$ 。可得信息对称情况下银行 利率的下限明显低于其在信息不对称情况下的值。

第二步：固定银行贷款利率 $r$, 并在此基础上比 较信息对称与信息不对称情况下银行对上游企业贷 款金额的区别。同样，让式(8)分别与不等式(7)和不 等式 (12) 结合, 经过整理和适当的放大和缩小后, 得 到如下不等式。

$$
I \leq \min \left\{\frac{\alpha}{B-\beta \Delta \theta}, \frac{[r-\beta(1-\Delta \theta)](1-\delta)}{\beta B}\right\}
$$

在利润最大化假设下, 激励约束变成紧的, 这个 时候在信息对称和信息不对称情况下的激励约束分 别变为 (15) 和 (16)

$$
\begin{gathered}
\alpha \beta\left[F\left(\alpha \mid I \theta_{L}\right)-F\left(\alpha \mid I \theta_{H}\right)\right]= \\
I(B-\beta \Delta \theta)+\beta\left[\int_{\alpha}^{I \theta_{H}} F\left(\tau \mid I \theta_{H}\right) d \tau-\int_{\alpha}^{I \theta_{L}} F\left(\tau \mid I \theta_{L}\right) d \tau\right](15) \\
\int_{I(1+r)}^{I \theta_{H}}[\tau-I(1+r)] f\left(\tau \mid I \theta_{H}\right) d \tau= \\
B I+\int_{I(1+r)}^{I \theta_{L}}[\tau-I(1+r)] f\left(\tau \mid I \theta_{L}\right) d \tau \quad(16)
\end{gathered}
$$

基于等式(15)和(16)本节做了数值分析, 研究了下 游违约概率 $p$ 、信息不对称 $\Delta \theta$ 和商业信用敞口 $\delta$ 对银行借贷利率 $r$ 和借贷金额 $I$ 的影响。

图 3 和图 4 是信息对称和信息不对称情况下, 贷 款利率与下游违约风险, 以及商业信用敞口的关系。 可以看出, 无论是信息对称还是不对称。下游违约风 险增加和商业信用敞口扩大都推高了贷款利率。

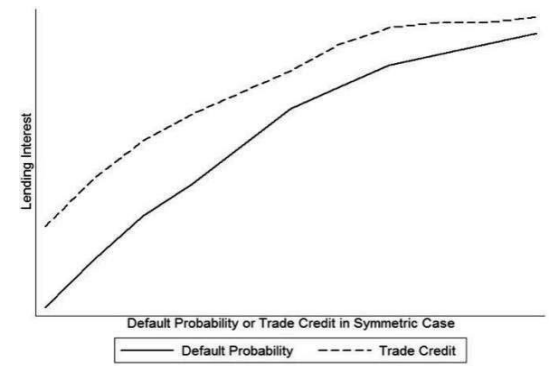

图 3: 信息对称情况下的贷款利率

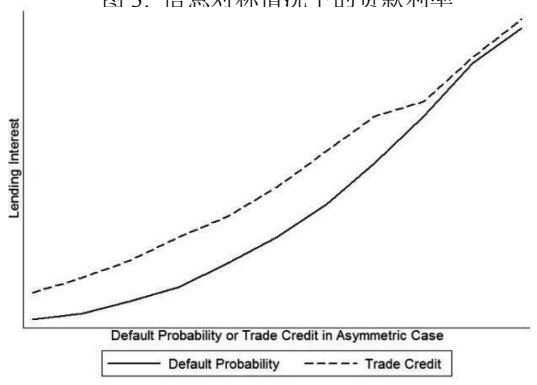

图 4 :信息不对称情况下的贷款利率

然后再考虑贷款金额, 银行对贷款金额 $I$ 的确 定就不像贷款利率 $r$ 那么简单了。贷款金额与买方 违约概率 $P$ 之间的关系, 以及贷款金额与商业信 用敞口的关系, 不能看作是参数 $P$ 和 $\delta$ 的单调递 减函数。图 5 表示的双峰关系表示了商业信用敞口 和贷款金额之间的关系, 它的形状与贷款金额和买 方的违约概率曲线相似, 意味商业信用敞口对借贷 金额的影响颇为复杂。

\section{3. 结束语}

本文刻画了供应链中商业信用和信息不对称如何 影响银行对上游企业的信贷策略, 在信息不对称情况 下构建了移动平均投资模型。结果表明, 银行为了最 大化自身利益而对上游借款者设置最优的贷款金额 和贷款利率, 此结果会促使上游借款人选择高努力水 
Risk Analysis and Crisis Response in Big Data Era (RAC-16)

平，并满足上游企业激励相容约束. 通过比较信息对

称与信息不对称下的激励相容约束, 证实了信息不对 称将使银行通过提高贷款利润来弥补可能的损失，从 而加大了上游企业融资成本。由于商业信用所带来的 潜在风险, 当下游企业的违约倾向增加时, 上游企业 的融资成本也会推高。同时, 商业信用的敞口变大, 也会促使银行贷款利率提高。然而, 无论是商业信用 或者是下游企业违约风险, 都不能单独影响信贷金 额。

为了简化理论框架, 突出信息不对称下银行贷款 策略的关键问题, 我们做了一些很重要的假设, 如银 行的完全竞争市场和上下游企业的议价能力, 而这样 的假设过于强烈。而供应链和商业信用合同的细节在 本文中没有关注。如果把这些考虑纳入我们的框架可 能会给供应链信贷策略研究带来更多的见解。此外, 由于供应链企业内部交互数据的大规模获取性困难, 文中的主要结论仅仅是形式化的推论，而缺乏分实证 支持, 通过经验数据对本文结论进行检验也将是未来 的进一步工作之一。

\section{参考文献}

[1] Y.F Li, Z.F. Zhou. Research on model for evaluating risks of venture capital projects. Journal of Risk Analysis and Crisis Response, 2001, 1(2): 142148.

[2] J.K. Yu, Z.F. Zongfang. The errors estimate of the multistage combined investment risk assessment. Journal of Risk Analysis and Crisis Response, 2001, 1(2):106-109.

[3] N. Wilson, B. Summers. Trade credit terms offered by small firms: survey evidence and empirical analysis. Journal of Business Finance \& Accounting, 2002, 29(4): 317-351.

[4] R. Fishman, I. Love. Trade Credit, financial intermediary development and industry growth. Journal of Finance, 58(1): 353-373,2003.

[5] P. Csoka, D. Havran, N. Szucs. Corporate financing under moral hazard and the default risk of buyers. Central European Journal of Operation Research, 2013, 62(10):1-16

[6] M.A. Peterson, R.G. Rajan. Trade credit: theories and evidence. The Review of Financial Studies, 1997, 3(10): 661-691.

[7] R. Albuquerque, T. Ramadoral, S.W. Watugala. Trade credit and cross-country predictable firm retures. Journal of Financial Economics,2015, 3(115): 596-613.

[8] M. Psillaki, K. Eleftheriou. Trade credit, bank credit, and fight to quality: evidence from French SMEs. Journal of Small Business Management, 2015, 4(53): 1219-1240.

[9] P. Kouvelis, W. Zhao. Financing the newsvendor: supplier vs. bank, and the structure of optimal credit contracts. Operations Research, 2016, 3(60): $566-580$

[10] G. Lai, L.G. Debo, K. Sycara. Sharing inventory risk in supply chain: the implication of financial constraint. Omega, 2009, 37(4):811-825.

[11] M. Dada, Q.J. Hu. Financing newsvendor inventory. Operations Research Letters,2008, 36(5): 569-573.

[12] E. Koskela, R. Stenbacka. Is there a tradeoff between bank competition and financial fragility. Journal of Banking \& Finance, 2000, 8 24(12): 1853-1873.

[13] J. Tirole. The Theory of Corporate Finance. Princeton University Press, Princeton, 2006. 